\title{
HEALTHY AGING - THE ROLE OF SPORT SCIENCE
}

\author{
Nenad Stojiljković, Milovan Bratić, Saša Pantelić \\ University of Nis, SERBIA
}

\begin{abstract}
Summary
Introduction: Rapid technical and technological development make our lives easier and more comfortable but significantly decrease the level of physical activity which is extremely important in preserving health related skills and keeping ability to move even during the old age. The purpose of this paper is to present the health benefits of sports science research on the process of aging and to summarize what is done and what should be done in the field of sports science related to healthy aging.

Methodology: For the purpose of this review article literature search was conducted using the following bases: MEDLINE, Google Scholar, Kobson and DOAJ. The selection was based on criteria related to age which participants belong (60-80 years), then that study was related to the influence of sports science on the components of physical fitness, health, body composition and anthropometric parameters in elderly.

Results: Aging is a complex process which include many variables such as genetics, lifestyle, chronic disease, environment, etc. Research within the sports science continuously prove the health benefits of exercise (both aerobic and anaerobic) with older population and improving their strength, power, flexibility, body composition, independence in daily activities.
\end{abstract}

Conclusion: One of the main factors in the process of healthy aging is exercise. Different exercise programs based on the evidence from sport science could improve physical fitness of elderly.

Key words: exercise, older, aging, science, physical activity

\section{Introduction}

Rapid technical and technological development make our lives easier and more comfortable but significantly decrease the level of physical activity which is extremely important in preserving health related skills and keeping ability to move even during the old age. Recent researches prove the importance of physical activity for older and statement that some physical activity is better than none and that any amount of physical activity can gain some health benefits (Chodzko-Zajko, 2009). Aging is inevitable process which is not fully discovered and explained, especially biological nature of aging and underpinning physiological processes. According to United Nations the older age starts over 60, but the newest definitions of older ages do not use ages as criteria but some kind of limitations in performing daily activities and the loss of previous roles. Therefore, it is possible to move the limit of the old ages. This is especially important if we know that in the developed world people are living longer than ever before. Life expectancy is increasing by two years every decade which means that most of western countries will have predominantly older population. Aging is traditionally linked with disease and frailty, but the recent studies gave the evidences that it is not necessary and that we can save a lot of our health related fitness, as well as skill related fitness by the well planned exercise program. Planning physical activity of older adults is very challenging because we usually have the presence of different chronic disease and physical limitations. More than $80 \%$ of older people have at least one chronic health problem such as cardiovascular disease, cancer, diabetes, osteoporosis, sarcopenia, arthritis. The issue of aging is very complex and in the focus of different scientific disciplines (medicine, physiology, gerontology, biology, andragogy). In some cases, researches in the field of aging resulted by the new specific disciplines of science such as biogerontology. In last few decades' problem of aging became very important issue for the research within the sport science (human movement science).

The purpose of this paper is to present the health benefits of sports science research on the process of aging and to summarize what is done and what should be done in the field of sports science related to healthy aging. Understanding the role of exercising in slowing down the aging related changes in health and functions is important aspect of public health. 


\section{Methodology}

For the purpose of this review article literature search was conducted using the following bases: MEDLINE, Google Scholar, Kobson and DOAJ. The selection was based on criteria related to age which participants belong (60-80 years), then that study was related to the influence of sports science on the components of physical fitness, health, body composition and anthropometric parameters in elderly.

\section{Results and discussion}

Aging is a complex process which include many variables such as genetics, lifestyle, chronic disease, environment, etc. Research within the sports science continuously prove the health benefits of exercise (both aerobic and anaerobic) with older population and improving their strength, power, flexibility, body composition, independence in daily activities.

There is an extensive volume of researches in sports science dedicated to the search for the fundamental physiological, genetic and biomechanical processes which are in the basis of the detrimental of functional integrity.

According to the researches in the sports science physical abilities in later life are compliant to improvement. Positive relationship between physical activity and improvements in physical fitness in older people is the crucial in slowing down the process of aging. Based on the published articles we divide the influence of sport science on different aspects of human life.

Physical activity, aging and musculoskeletal system Decreasing of muscle function in the older ages is caused by numerous factors which has to be identified to reduce such loss. Muscle weakness is an independent risk factor for high mortality in older adults (Metter, Talbot, Schrager, and Conwit, 2002). Older adults with low muscle strength have a 2.6fold greater risk of severe mobility limitation, 4.3fold greater risk for slow gait speed, and 2.1-fold greater risk of mortality compared to older adults with high muscle strength (Manini et al, 2007). One of the main factors of losing muscle strength in older is sarcopenia. This is the age associated loss of skeletal muscle mass which causes reduction of physical strength and ability to perform activities of daily living (Thompson, 2007, Pantelic et al., 2014). In that manner older loose the independence and their quality of life is seriously diminished. The loss of independence is related to high economic healthcare burden (Thompson, 2007) and it became one of the main problem for the state financial system. Today, there is no effective and safe therapy of sarcopenia and related loss of physical fitness. Current standard in care of sarcopenia is appropriate nutrition and physical activity (Phu, Boersma, and Duque, 2015). Exercise improves muscle mass through the activation of the satellite cell differentiation and activity. The role of satellite cells is crucial because it has been hypothesized that the only way to increase the synthesis of myofibrilar protein is through the incorporation of new nuclei from satellite cells into the parent cell (Thornel, 2011). During exercise active muscles release inflammatory and hormonal substances (cytokines and growth factor) that are satellite cell stimulants. These factors determine the activation of the satellite cells (Kang \& Kraus, 2010).

Researches related to exercise and sarcopenia show that not any kind of exercise is good prevention against sarcopenia. Endurance exercise program increase maximal oxygen consumption by improving the central and peripheral circular capacities (increased muscular capillarity, increased stroke volume, expanded number of mitochondria, greater activity of mitochondrial enzymes) (Bassett \& Howley, 2000), but in the fight against sarcopenia the most effective program is resistance exercise. This kind of exercise impact fiber-cross sectional area due to an increase in the number of myofibrils with the fast-twitching fiber types being responsible for the most increase in muscle size (Landi et al., 2014; Kang \& Kraus, 2010).

One study shows that although the loss of muscle mass is associated with the decline in strength in older adults, this strength decline is much more rapid than the concomitant loss of muscle mass, suggesting a decline in muscle quality. Moreover, maintaining or gaining muscle mass does not prevent aging-associated declines in muscle strength (Goodpaster et al., 2006). The authors concluded that in addition to muscle quantity, muscle quality may be an important determinant of loss of strength with aging.

\section{Physical activity, aging and cardiovascular system}

Age related changes cause structural degeneration of the cardiovascular system which lead to the func- 
tional decline. The causes of changes are very complex and diversified (Ferrari, Radaelli, \& Centola, 2003). This changes are not necessary related to disease and might be developed even in the absence of any disease. Moreover, it is very difficult to make a difference between age-dependent alterations and changes caused from disease conditions (atherosclerosis, heart failure, and diabetes mellitus) (Ferrari, 2002). Aging do not cause only decline in cardiovascular function. Some evidence proves that in elderly some features of cardiovascular system paradoxally increase (mild increase in heart weight, left ventricular hypertrophy, increased dimensions of cardiomyocites). Such changes cause alterations in functions of cardiovascular system. The heart becomes slightly hypertrophic and hyporesponsive to sympathetic stimuli which is especially important for exercise because the heart and myocardial contractility do not response with adequate intensity like in young age. Exercise induced increases in heart rate and myocardial contractility are less intensive in elderly. During the maximal effort peak cardiac output of elderly is lower by 20 to $30 \%$ than in young healthy subjects. This decrease is caused more by the inability of heart to increase heart rate than by the changes in stroke volume. During exercise myocardial contricle mainly engages the Frank-Starling mechanism which increases end-diastolic and end-systolic volume. Physiologist and cardiologists explain the alterations in cardiac function of elderly during exercise by the example of the younger heart subjected to beta blocker treatment (Julius et al., 1967).

Recent studies prove that regular vigorous exercise is probably the most powerful treatman for cardiovascular health even in older adults. Study of 416000 adults followed for a mean of 8 years, 40 to 50 minutes per day of vigorous exercise reduced risk of death by about $40 \%$ (O'keefe \& Lavie, 2012). Light to moderate physical activity reduced death rates too, albeit not as strongly, but in this case more physical activity appeared to be better, with no plateau out to 110 min daily (O'keefe \& Lavie, 2012). Studies performed on elite athletes proved benefits of vigorous exercise on healthy aging and longevity. Cross-country ski racers lower cardiovascular disease risk by $57 \%$ and overall mortality by $58 \%$ (Farahmand et al., 2003). Also, study with veteran athletes with arrhythmias shows that participants have higher heart rate variability which is considered as cardioprotective effect (Jensen-Urstad, 1998).
Aging causes different alterations in vascular system too. With ages large arteries become elongated and their lumen become enlarged and wall thickened. The greater age-related change in vascular function, particularly in arterial function, is impairment of distensibility and cushioning function of the aorta and its major branches (Roach, \& Burton, 1959). Changes are not solely dependent on structural changes in vascular system, but also is affected by humoral and endothelian regulation of vascular smooth muscle tone (aged vessels show an increased endothelial permeability and a reduced nitric oxide-dependent vasodilator response to acetylcholine (Taddei et al., 1995).

Physical activity has a significant impact on the structure of various blood vessels. These morphological changes are followed by functional changes and improving of blood flow. Physical activity induces angiogenesis which is an expansion of the capillary network by the formation of new blood vessels at the level of capillaries and resistance arterioreles, and arteriogenesis which is an enlargement of existing vessels (Leung et al., 2008). Exercise can cause also an anti-inflammatory effect in vascular tissue. This kind of exercise influence can be especially important in preventing atherosclerosis as an inflammatory disease that is mediated by monocyte derived macrophages which accumulate in arterial plaques and become activated to realize cytokines that cause tissue damage (Leung et al., 2008). Exercise can produce a short-term inflammatory response that is accompanied by leukocytosis, increase in oxidative stress, and plasma levels of CRP. This pro-inflammatory response is followed by a long term anti-inflammatory response. Exercise decrease levels of CRP, IL- 6 and TNF- $\alpha$ and increases levels of anti-inflammatory substances such as IL-4 and IL-10 (Kasapis, \& Thompson, 2005; Plaisance, \& Grandjean, 2006)

\section{Physical activity, aging and brain health}

One of the causes of aging is declining of the cortical volume of prefrontal brain regions and detrimented executive functioning. The question that sports scientist consider is "Can we protect the anatomical structures and functions of our brain by the physical activity?" Recent studies (Marks et al., 2007) proved positive relationship between higher aerobic fitness (VO2max) and improved executive functioning in older adults and their functional anatomy of the brain. With the ages the integrity of white matter 
decline, but the aerobic exercise may be related to differing levels of white matter integrity which may in turn cause preserving of cognitive function.

Several studies suggest that higher physical activity levels, maximal aerobic capacity and aerobic activities are in high correlation with preservation of cognitive function and decrease of Alzheimer disease (Beydoun et al., 2014; Barnes et al., 2003).

Resistance training also can preserve cognitive functions and prevent development of cognitive impairment. Study from 2017 (Mavros et al., 2017) about the influence of different strength training programs on cognitive functions prove some new benefits of resistance training. They revealed evidence that progressive resistance training significantly improve strength in healthy elderly adults and also increase maximal oxygen consumption. Also, they revealed strong relationship between the magnitude of strength gain and cognitive benefits which explain the influence of resistance training on cognition. The explanation of this very new findings could be in the relationship between deficiency of insuline-like growth factor 1 (IGF-1) and cognitive dysfunction and incident dementia in older adults. Resistance training program increase IGF-1 which can lead to the improvements of cognitive functions. The second explanation of this relationship could be increases in brain-derived neurotrophic factor (BDNF) after 12 months walking program and improved function of brain. Exercise has influence on the level of cortisol. High cortisol levels are associated with worse cognitive function, small brain volume in older adult's memory impairment and atrophy of hippocampi. Resistance training can decrease basal cortisol level and make benefits in cognitive functions.

\section{Conclusion}

One of the main factors in the process of healthy aging is exercise. Numerous researches demonstrate a strong relationship between exercise and protection of the most important body systems such as musculoskeletal, cardiovascular and nervous system. Different exercise programs based on the evidence from sport science could improve physical fitness of elderly. This evidences could encourage sports scientists to work on development of wide range of exercise programs from calisthenics and resistance training to pure endurance training program. Influence of exercise (resistance or endurance training) on brain and nervous system is still not fully discovered. Therefore, one of the following and very important tasks of sport science is the researches of the protection of brain and nervous system by different types of exercise.

\section{References:}

Barnes, D. E., Yaffe, K., Satariano, W. A., \& Tager, I. B. 2003. A longitudinal study of cardiorespiratory fitness and cognitive function in healthy older adults. Journal of the American Geriatrics Society, 51(4), pp.459-465.

Bassett Jr, D.R. and Howley, E.T., 2000. Limiting factors for maximum oxygen uptake and determinants of endurance performance. Medicine \& Science in Sports \& Exercise, 32(1), p.70.

Beydoun, M. A., Beydoun, H. A., Gamaldo, A. A., Teel, A., Zonderman, A. B., \& Wang, Y. 2014. Epidemiologic studies of modifiable factors associated with cognition and dementia: systematic review and meta-analysis. BMC public health, 14(1), pp.643.

Chodzko-Zajko, W.J., Proctor, D.N., Singh, M.A.F., Minson, C.T., Nigg, C.R., Salem, G.J. and Skinner, J.S., 2009. Exercise and physical activity for older adults. Medicine \& science in sports \& exercise, 41(7), pp.1510-1530.

Farahmand, B. Y., Ahlbom, A., Ekblom, Ö., Ekblom, B., Hållmarker, U., Aronson, D., \& Brobert, G. P. 2003. Mortality amongst participants in Vasaloppet: a classical long-distance ski race in Sweden. Journal of internal medicine, 253(3), pp.276-283.

Ferrari, A.U., 2002. Modifications of the cardiovascular system with aging. The American journal of geriatric cardiology, 11(1), pp.30-34.

Ferrari, A.U., Radaelli, A. and Centola, M., 2003. Invited review: aging and the cardiovascular system. Journal of Applied Physiology, 95(6), pp.2591-2597.

Goodpaster, B.H., Park, S.W., Harris, T.B., Kritchevsky, S.B., Nevitt, M., Schwartz, A.V., Simonsick, E.M., Tylavsky, F.A., Visser, M. and Newman, A.B., 2006. The loss of skeletal muscle strength, mass, and quality in older adults: the health, aging and body composition study. The Journals of Gerontology Series A: Biological Sciences and Medical Sciences, 61(10), pp.1059-1064.

Jensen-Urstad, K., Bouvier, F., Saltin, B., \& Jensen-Urstad, M. 1998. High prevalence of arrhythmias in elderly male athletes with a lifelong history of regular strenuous exercise. Heart, 79(2), pp.161-164.

Julius, S., Amery, A., Whitlock, L. S., \& Conway, J. 1967. Influence of age on the hemodynamic response to exercise. Circulation, 36(2), pp.222-230.

Kang, J.S. and Krauss, R.S., 2010. Muscle stem cells in developmental and regenerative myogenesis. Current opinion in clinical nutrition and metabolic care, 13(3), p.243.

Kasapis, C., \& Thompson, P. D. 2005. The effects of physical activity on serum C-reactive protein and inflammatory markers: a systematic review. Journal of the American College of Cardiology, 45(10), pp.1563-1569.

Landi, F., Marzetti, E., Martone, A.M., Bernabei, R. and Onder, G., 2014. Exercise as a remedy for sarcopenia. Current Opinion in Clinical Nutrition \& Metabolic Care, 
17(1), pp.25-31.

Leung, F. P., Yung, L. M., Laher, I., Yao, X., Chen, Z. Y., \& Huang, Y. U. 2008. Exercise, vascular wall and cardiovascular diseases. Sports Medicine, 38(12), pp.1009-1024.

Manini, T.M., Visser, M., Won-Park, S., Patel, K.V., Strotmeyer, E.S., Chen, H., Goodpaster, B., De Rekeneire, N., Newman, A.B., Simonsick, E.M. and Kritchevsky, S.B., 2007. Knee extension strength cutpoints for maintaining mobility. Journal of the American Geriatrics Society, 55(3), pp.451-457.

Marks, B.L., Madden, D.J., Bucur, B., Provenzale, J.M., White, L.E., Cabeza, R. and Huettel, S.A., 2007. Role of aerobic fitness and aging on cerebral white matter integrity. Annals of the New York Academy of Sciences, 1097(1), pp.171-174.

Mavros, Y., Gates, N., Wilson, G. C., Jain, N., Meiklejohn, J., Brodaty, H., ... \& Baker, M. K. (2017). Mediation of cognitive function improvements by strength gains after resistance training in older adults with mild cognitive impairment: outcomes of the study of mental and resistance training. Journal of the American Geriatrics Society, 65(3), pp.550-559.

Metter, E.J., Talbot, L.A., Schrager, M. and Conwit, R., 2002. Skeletal muscle strength as a predictor of all-cause mortality in healthy men. The Journals of Gerontology Series A: Biological Sciences and Medical Sciences, 57(10), pp.B359-B365.

O'keefe, J. H., \& Lavie, C. J. 2012. Run for your life... at a comfortable speed and not too far. Heart, 99(8), pp.516-519

Pantelic, S., Kostic, R., Popovic, R. Aleksandrovic, M.,
Uyunovic, S., Milanovic, Z., Jorgic, B., \& Stojiljkovic, N. 2014. Physical activity and fitness components in elder$l y$ (in Serbian). The Ministry of Education, Science and Technological Development of the Republic of Serbia; Nis: Faculty of sport and physical education

Phu, S., Boersma, D. and Duque, G., 2015. Exercise and sarcopenia. Journal of Clinical Densitometry, 18(4), pp.488-492.

Plaisance, E. P., \& Grandjean, P. W. 2006. Physical activity and high-sensitivity C-reactive protein. Sports Medicine, 36(5), pp.443-458.

Roach, M. R., \& Burton, A. C. 1959. The effect of age on the elasticity of human iliac arteries. Canadian journal of biochemistry and physiology, 37(4), pp.557-570.

Taddei, S., Virdis, A., Mattei, P., Ghiadoni, L., Gennari, A., Fasolo, C. B., ... \& Salvetti, A. 1995. Aging and endothelial function in normotensive subjects and patients with essential hypertension. Circulation, 91(7), pp.19811987.

Thompson, D.D., 2007. Aging and sarcopenia. Journal of Musculoskeletal and Neuronal Interactions, 7(4), p.344.

Thornell, L.E., 2011. Sarcopenic obesity: satellite cells in the aging muscle. Current Opinion in Clinical Nutrition \& Metabolic Care, 14(1), pp.22-27.

\section{Name and address of the corresponding author}

Dr Nenad Stojiljkovic, assistance professor

Address: Faculty of Sport and Physical Education, Carnojevica 10a, 18000 Nis, Serbia

E mail: snesadif@yahoo.com

Mobile: +381638084961 